\title{
Radio resource reservation for heterogeneous traffic in cellular networks with spectrum leasing
}

\author{
Show-Shiow Tzeng ${ }^{1 *}$, Ying-Jen Lin² and Yu-Ching Hsu ${ }^{3}$
}

\begin{abstract}
Radio channel reservation is used to alleviate call dropping which may occur in two situations: (i) hand-off between cells in cellular networks, and (ii) channel withdrawal in wireless networks with spectrum leasing. In this article, we study a radio resource reservation scheme for heterogeneous traffic in a cellular network with spectrum leasing, in which one reservation pool is used to alleviate the two types of call droppings. Since different types of traffic have different tolerances to the exhaustion of channels, it is critical for different types of traffic to select the optimal size of the reservation pool such that the channel requirements of different types of traffic are satisfied while throughput is maximized. A three-dimensional Markov chain is presented to find the optimal size of reservation pool. Numerical and simulation results show that (i) the selected parameters of reservation satisfy the quality-of-service requirements of different types of traffic while produce high throughput, and (ii) channel withdrawal yields higher impact on real-time traffic than non-real-time traffic in terms of throughput.
\end{abstract}

\section{Introduction}

Radio resource is scarce and precious; however, radio spectrum is underutilized in most wireless systems in which radio spectrum is statically assigned [1]. One possible way to efficiently utilize the radio spectrum is to allow spectrum sharing between various wireless systems [2-6]. One wireless system can lease radio spectrum from (or out to) another wireless system. Then, mobile users in one system can dynamically access radio channels in another one. In such an environment, a system that leases out its radio channels to another always has the first priority to use its radio channels; that is, the system can withdraw its radio channels from another when the system requires the radio channels.

An ongoing call may be dropped by channel withdrawal. When a channel is forcibly withdrawn from a mobile user, the mobile user releases the withdrawn channel and attempts to hand-off to another idle channel in order to continue its communication. If no free channel is available to the mobile user, the user is dropped; otherwise, the user

\footnotetext{
*Correspondence: sstzeng@nknucc.nknu.edu.tw

${ }^{1}$ Department of Optoelectronics and Comm. Engineering, National Kaohsiung Normal University, Kaohsiung, 802 Taiwan

Full list of author information is available at the end of the article
}

continues its communication. The probability that a handoff user due to channel withdrawal is dropped is called withdrawal dropping probability herein. To reduce the call dropping, a number of channels are reserved for the calls suffering channel withdrawal [7].

Cellular wireless networks are a category of widelydeployed wireless systems, in which service areas consist of cells. In such a wireless network, mobile users may move from one cell to another. When a mobile user moves from a cell to a neighbor cell, a inter-cell hand-off procedure is initiated to continue the mobile user's communication. If free channels in the neighbor cell are insufficient to satisfy the channel requirement of the mobile user, the mobile user is dropped. The probability that an inter-cell hand-off attempt fails is referred to as inter-cell hand-off dropping probability in this article. From the viewpoint of mobile users, the inter-cell hand-off dropping probability should be as low as possible. To provide mobile users with low inter-cell hand-off dropping probability, threshold based channel reservation schemes have been presented in $[8,9]$.

This article considers a cellular network which can lease a spectrum band from another wireless system; in such a network, traffic congestion can be alleviated by using

\section{Springer}

C 2012 Tzeng et al: licensee Springer. This is an Open Access article distributed under the terms of the Creative Commons Attribution License (http://creativecommons.org/licenses/by/2.0), which permits unrestricted use, distribution, and reproduction in any medium, provided the original work is properly cited. 
spectrum leasing. As mentioned in previous paragraphs, channel reservations have been separately reserved for the two types of call dropping which respectively result from channel withdrawal and inter-cell hand-off. Due to the gain of resource sharing [10], the two reservations can be implicitly combined into a reservation pool [11]. However, the optimal reservation in the pool is not the sum of the two optimal reservations in $[7,8]$, which reasons are as follows. First, the optimal reservation in [8] is found in cells with fixed channels, but the cells considered herein have variable channels due to channel leasing and withdrawal, which complicates the channel reservation for inter-cell hand-off calls. Second, the optimal reservation in [7] is found in an environment without inter-cell hand-off call arrivals, but the cellular network herein includes the intercell hand-off arrivals, which worsens the negative effect of channel withdrawal. Third, due to resource sharing, fewer channels are possibly reserved to keep the same level of dropping probability for the two types of call dropping. In summary, the penalty for the resource sharing gain is to increase the complexity of selecting the optimal number of reservation channels.

The selection of optimal reservation in the pool is further complicated for heterogeneous traffic which includes real-time and non-real-time traffic, because real-time and non-real-time traffics have different tolerances to the exhaustion of channels. Non-real-time traffic is moderately sensitive or insensitive to the exhaustion of channels; that is, when a channel is forcibly withdrawn from a nonreal-time user, the non-real-time user can be temporarily placed into a queue to wait a free channel in order to resume its communication. On the contrary, real-time traffic is sensitive to the exhaustion of channels; when a channel is forcibly withdrawn from a real-time user, the real-time user is dropped if no free channel is available to the real-time user.

Based on the descriptions in the above two paragraphs, it is critical and complicated to reserve the optimal number of channels in a single reservation pool such that

- the withdrawal dropping probabilities of real-time and non-real-time calls are guaranteed,

- the inter-cell hand-off dropping probabilities of real-time and non-real-time calls are kept below a certain level,

- the queuing (or waiting) time of non-real-time calls in a cell is guaranteed,

- the throughput (i.e. the completed calls per time unit) is maximized.

In this article, we present a threshold-based channel reservation scheme, which reserves channels in a single pool, for real-time and non-real-time traffic. A threedimensional Markov chain is developed to describe the system state of the channel reservation in a cellular wireless network with spectrum leasing. Based on the Markov chain, we calculate the desired quality-of-service metrics (in terms of the inter-cell hand-off dropping probability, withdrawal dropping probability and waiting time) and throughput. Then, given two thresholds for real-time and non-real-time traffic, we can apply our analyses to calculate the corresponding quality-of-services and throughput. Therefore, we can select optimal thresholds from a wide range of combinations of different thresholds for the threshold-based channel reservation such that the qualityof-services of mobile users are satisfied while throughput is maximized. Numerical and simulation results show that, the selected thresholds can guarantee the qualityof-service requirements of real-time and non-real-time traffic while produce high throughput.

The rest of this article is organized as follows. Section "Channel reservation in an environment of spectrum leasing" describes the threshold-based channel reservation in a cellular network with spectrum leasing. Section "Numerical analyses" describes our numerical analyses of the channel reservation scheme. Subsequently, performance evaluation is described in Section "Performance evaluation". Finally, some concluding remarks are presented in Section "Conclusions".

\section{Channel reservation in an environment of spectrum leasing} In this section, we first describe a cellular environment of spectrum leasing. Then, in such an environment, we describe a threshold based channel reservation scheme for real-time and non-real-time traffic.

\section{The environment of spectrum leasing}

A cellular wireless network may be licensed for holding a radio spectrum over a long period of time. The licensed radio spectrum can be further divided into radio channels. The licensed radio channels in a cellular wireless network are called "licensed channels" herein. After mobile users register in a cellular wireless network, the mobile users can use the licensed channels in the cellular wireless network. In addition, when the mobile users are using the licensed channels in the cellular wireless network, the cellular network does not forcibly withdraw the licensed channels from the mobile users. Although a mobile user may request one or more channels, we assume, for simplicity, that a mobile user merely requires one channel in this article.

A cellular wireless network can lease its licensed channels out to another wireless network. In this article, a wireless network that leases out its licensed channels is referred to as "channel licensee". A cellular network that leases radio channels from a channel licensee is referred to as "channel leaseholder". For a channel leaseholder, the radio channels that are leased from a channel licensee are called "leasehold channels". In a channel leaseholder, a 
leasehold channel can be allocated to a mobile user that registers in the channel leaseholder.

A leasehold channel in a channel leaseholder will be withdrawn when the channel licensee of the channel requires the channel. If this channel withdrawal occurs, the mobile user which is using the leasehold channel will perform hand-offs between different spectrums that will be described in the following subsection. Besides, the channel is held and used by the channel licensee of the channel. When the channel licensee releases the channel, the channel becomes free and is available to the channel leaseholder of the channel.

In this article, we consider a cellular wireless network which can lease channels from another wireless network. In the network, there are licensed channels and leasehold channels in a cell. Due to channel withdrawal, the channels available to mobile users in a cell are the licensed channels in the cell plus the leasehold channels which are not withdrawn in the cell. Note that the licensed channels in a cell could be leasehold channels in cells on another network. Since the cell has the first priority to use the licensed channels, the cell could withdraw the license channels that are used by the cells on another network, which can be regarded as that the licensed channels, which are used or not used by another network, are always available to the cell. Therefore, the scenario that the licensed channels in a cell are leased out to the cells on another network is implicitly included in our considered environment.

\section{Threshold based channel reservation}

Two thresholds $T_{r}$ and $T_{n}$ are separately used to prevent new real-time and non-real-time mobile users from entering a congested cell to consume a free channel, worsen a congested situation and degrade quality-of-services. The thresholds are applied to new mobile users but are not applied to those ongoing mobile users suffering intercell hand-off or channel withdrawal. In other words, the thresholds reserve a number of free channels for those ongoing mobile users to continue their communications. Under such a threshold based reservation architecture, call admission, inter-cell hand-off and channel withdrawal procedures are described in detail as follows.

Call admission procedure When a new mobile user arrives at a cell, a call admission control procedure is initiated to determine whether to accept the user. When a new real-time mobile user arrives at a cell, the real-time user is admitted into the cell if the number of real-time mobile users in the cell is less than a threshold $T_{r}$ and there is at least one free channel in the cell; otherwise, the real-time user is blocked. When a new non-real-time mobile user arrives at a cell, the non-real-time user is admitted into the cell if the number of non-real-time mobile users in the cell is less than a threshold $T_{n}$ and there is at least one free channel in the cell; otherwise, the non-real-time user is blocked. Figure 1 shows the flow diagram of the above call admission control for new mobile users.

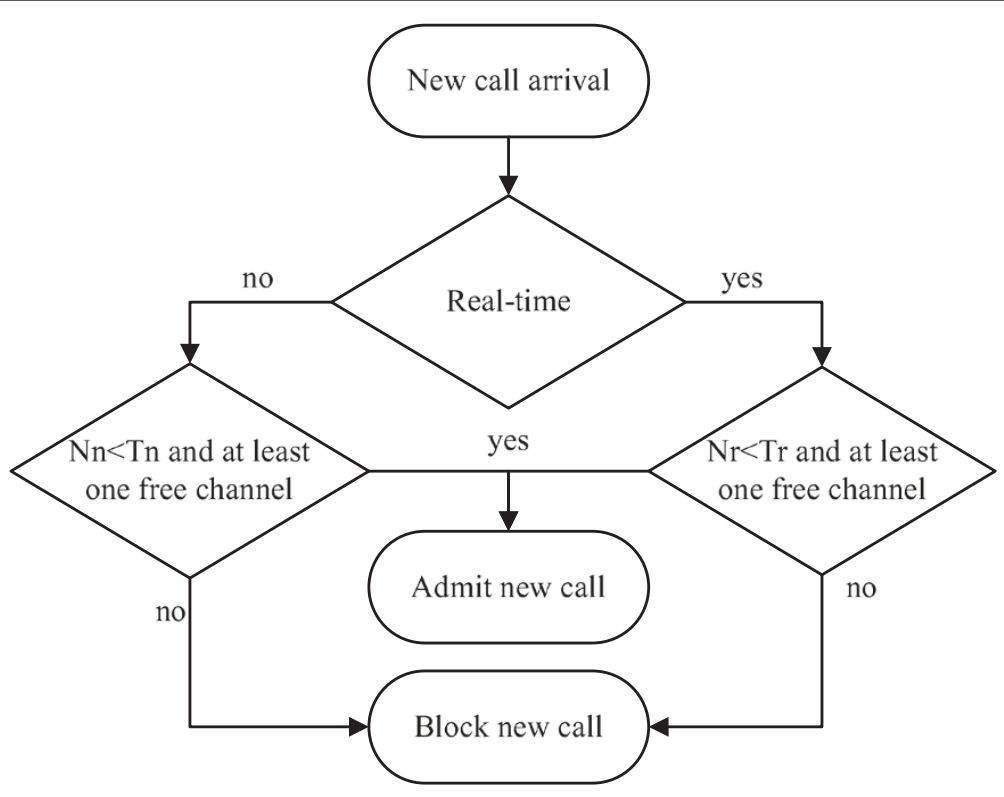

Nr: number of real-time users in a cell

Nn: number of non-real-time users in a cell

Figure 1 Call admission procedure. 
Inter-cell hand-off procedure Mobile users may handoff from one cell to another due to mobility. When an inter-cell hand-off call arrives at a cell, an inter-cell handoff procedure is initiated. If there is at least one free channel in the cell, the inter-cell hand-off call hand-offs successfully and continues its communication. Otherwise, the following operations are further performed according to the type of the inter-cell hand-off call.

- Since non-real-time users are insensitive to transmission delay, non-real-time users can tolerate pauses in data transmission. When there is no free channel in a cell, a non-real-time inter-cell hand-off call can be placed into a queue to wait for a free channel. If there is at least one free room in the queue, the non-real-time inter-cell hand-off call is placed into the queue; otherwise, the inter-cell hand-off call is dropped.

- If the inter-cell hand-off call is real-time, the following two conditions are checked: (i) whether one or more non-real-time users are using channels in the cell and (ii) whether the number of non-real-time users in a queue is less than $C_{q}$, where $C_{q}$ is the maximum number of mobile users which can be accommodated in the queue. If the two conditions are satisfied, one of the non-real-time users which are using channels is randomly selected, interrupted and placed into a queue; then, the channel occupied by the non-real-time user is released to the real-time inter-cell hand-off call. Otherwise, the real-time inter-cell hand-off call is dropped.
Figure 2 shows the flow diagram of the inter-cell handoff control procedure in the threshold based reservation scheme.

Channel withdrawal procedure When a channel withdrawal occurs, a free leasehold channel or a busy leasehold channel is withdrawn. If a free leasehold channel is withdrawn in a cell, mobile users in the cell do not be affected and continue their communications. If a busy leasehold channel is withdrawn from a mobile user in a cell, the mobile user will perform the following procedure, which is divided into three cases. Case I: if there is at least one free channel, the mobile user hand-offs from the withdrawn channel to the free channel. Case II: if there is no free channel and the mobile user is non-real-time user, the status of the queue in the cell is checked. If the queue is full, the non-real-time user is dropped; otherwise, the non-real-time user is placed into the queue to wait for a free channel. Case III: if there is no free channel and the mobile user is real-time user, the following two conditions are checked: (i) whether one or more non-real-time users are using channels in the cell, and (ii) whether there is at least one room in the queue in the cell. If the two conditions are satisfied, one of the non-real-time users using the channels is randomly selected, preempted, and placed into the queue, and then the real-time user hand-offs from the withdrawn channel to the released channel; otherwise, the real-time user is dropped. Figure 3 displays the flow diagram for handling channel withdrawal when a channel withdrawal request arrives.

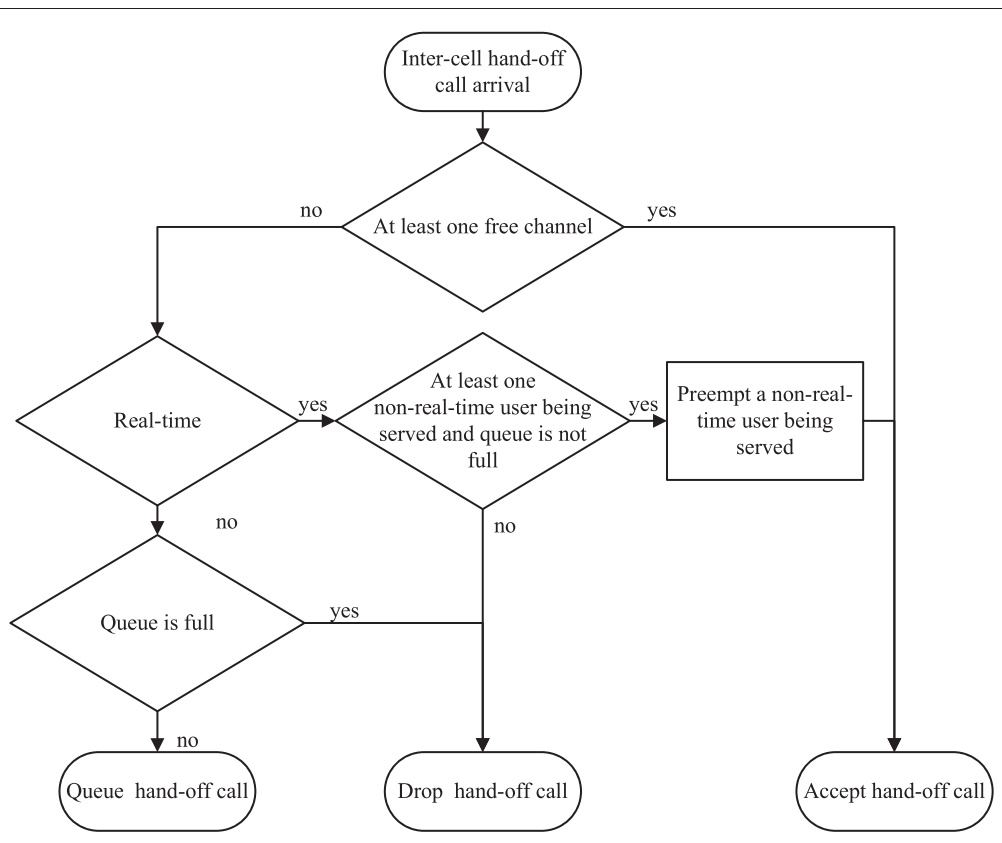

Figure 2 Inter-cell hand-off procedure. 


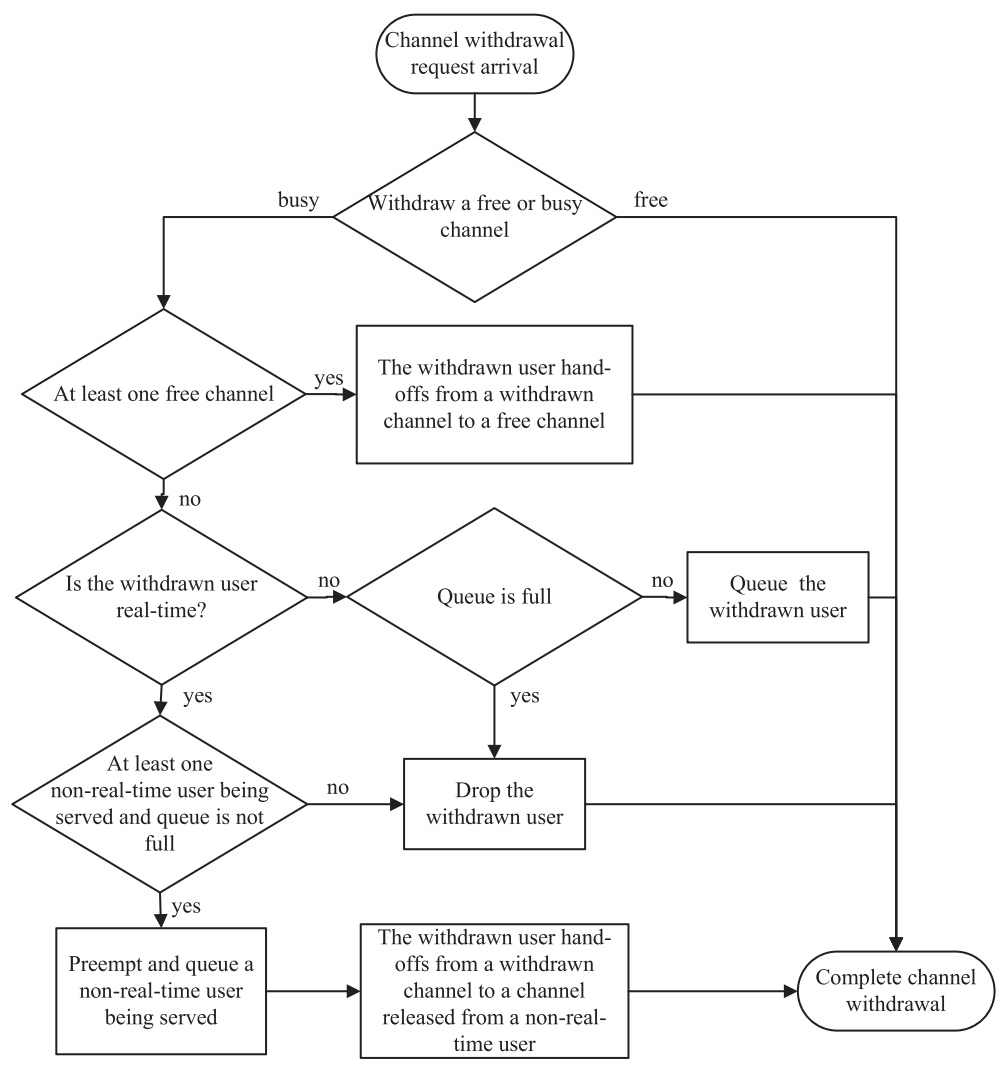

Figure 3 Channel withdrawal procedure.

When a withdrawal channel is released from a channel licensee, the channel is available to mobile users in a cell in a channel leaseholder. Moreover, when a mobile user completes its communication in a cell or moves out of a cell, a busy channel which is allocated to the mobile user is released and is also available to other mobile users in the cell. If there is at least one non-real-time user in a queue in a cell at the instant time of channel release, the channel will be allocated to a non-real-time user which is selected from the queue in a first-come-first-serve manner.

\section{Numerical analyses}

In this section, we first describe the assumptions used in our analyses. Then, we give an example to explain a threedimensional Markov chain which is used to analyze the performance of the threshold based reservation scheme. In order to formally express the global balance equations of the Markov chain, nine indicator functions are defined; then, an iterative procedure is introduced to solve the global balance equations. Finally, we derive the performance and quality-of-service metrics of the threshold based reservation scheme, and an procedure is introduced to obtain the performance metrics.

\section{Assumptions}

For tractable analysis and low computation complexity [12], we consider a cellular wireless system with homogeneous cells in this article. The radio channels in the cellular wireless network consist of licensed channels and leasehold channels. The total number of licensed channels in a cell is fixed and denoted by $C_{i}$; the maximum number of leasehold channels in a cell is fixed and denoted by $C_{e}$. Then, the maximum number of channels available in a cell, which is denoted by $C$, is equal to $C_{i}+C_{e}$.

New mobile users arrive at a cell according to a Poisson process with mean rate $\lambda$. Let $p_{r}$ be the probability that a new arrival is a real-time user. Then the probability that a new arrival is a non-real-time user is $1-p_{r}$. Let $\lambda_{r, n}$ and $\lambda_{n, n}$ be respectively the new arrival rates of realtime users and non-real-time users at a cell. Then, $\lambda_{r, n}=$ $\lambda p_{r}$, and $\lambda_{n, n}=\lambda\left(1-p_{r}\right)$. The lifetimes of a real-time call and a non-real-time call are exponentially distributed with means $\mu_{r, n}^{-1}$ and $\mu_{n, n}^{-1}$. The arrival of inter-cell handoff users into a cell is assumed to be a Poisson process. The mean rates of real-time and non-real-time inter-cell hand-offs are respectively denoted by $\lambda_{r, h}$ and $\lambda_{h, h}$, and the values of $\lambda_{r, h}$ and $\lambda_{n, h}$ are $\alpha \lambda_{r, n}$ and $\beta \lambda_{n, n}$, where $\alpha>0$ and $\beta>0$. The durations that a real-time user and a nonreal-time user stay in a cell are exponentially distributed 
with means $\mu_{r, h}^{-1}$ and $\mu_{n, h}^{-1}$ respectively. We assume that the arrival of channel withdrawals is a Poisson process with mean rate $\lambda_{w}$. The duration that leasehold channels are withdrawn is exponentially distributed with mean $\mu_{w}^{-1}$.

\section{The Markov chain for the threshold based channel reservation}

We use a three-dimensional Markov chain to analyze the performance of the threshold based channel reservation. For an example, the state transition rate diagram for the channel reservation with parameters $C_{i}=1, C_{e}=1$, $C_{q}=1, T_{r}=1$ and $T_{n}=1$ is shown in Figure 4 . Each of the states in the Markov chain is denoted by $(i, j, k)$, where $i$ denotes the number of real-time users in a cell, $j$ denotes the number of non-real-time users in a cell, and $k$ denotes the number of channels withdrawn from a cell. The possible value of $k$ is an integer which is greater than or equal to 0 but is less than or equal to $C_{e}$. Given $k$ channels are withdrawn from a cell, the possible value of $i$ is an integer which is greater than or equal to 0 but is less than or equal to $C-k$, and the possible value of $j$ is an integer which is greater than or equal to 0 but is less than or equal to $C+C_{q}-k$.

According to the call admission, inter-cell hand-off and channel withdrawal procedures in Section "Channel reservation in an environment of spectrum leasing", the transition rates between the states in the Markov chain are given on the arrows in Figure 4. Some transition rates are intuitive; parts of the transition rates are further explained as follows:

- The transition rates from state $(1,2,0)$ to states $(1,1,1)$ and $(0,2,1)$ are $0.5 \lambda_{w}$ because a leasehold channel is randomly withdrawn from a being served real-time user or a being served non-real-time user in state $(1,2,0)$.

- The transition rate from state $(0,1,0)$ to state $(0,2,0)$ is $\lambda_{n, h}$ because new non-real-time users are blocked due to the threshold $T_{n}=1$; similar phenomena also appear in states $(i, j, k)$, where $j \geq 1$.

- The transition rate from state $(1,0,0)$ to state $(2,0,0)$ is $\lambda_{r, h}$; this is because new non-real-time users are blocked due to the threshold $T_{r}=1$; similar phenomena are also in states $(i, j, k)$, where $i \geq 1$.

- The transition rate from state $(0,3,0)$ to state $(0,2,0)$ is $2 \mu_{n, n}+3 \mu_{n, h}$. Since two non-real-time users are being served in state $(0,3,0)$, the rate at which non-real-time users complete their communication is equal to $2 \mu_{n, n}$. Due to the mobility of mobile users, the rate at which mobile users hand-off to neighbor cells in state $(0,3,0)$ is $3 \mu_{n, h}$. Therefore, the total departure rate in state $(0,3,0)$ is $2 \mu_{n, n}+3 \mu_{n, h}$.

From the Markov chain in Figure 4, we can easily write down its corresponding global balance equations which general form is formally and concisely expressed in the Equation (10). Before concisely expressing the general form of the global balance equations of the Markov chain for the threshold based reservation scheme, we first define nine indicator functions.

Indicator functions To concisely present the balance equations, we first define an eligible state and nine indicator functions. A state $(i, j, k)$ is eligible if the following four conditions are satisfied, i.e. $0 \leq i \leq C, 0 \leq j \leq C+C_{q}$, $0 \leq k \leq C_{e}$ and $0 \leq i+j+k \leq C+C_{q}$. Given an eligible

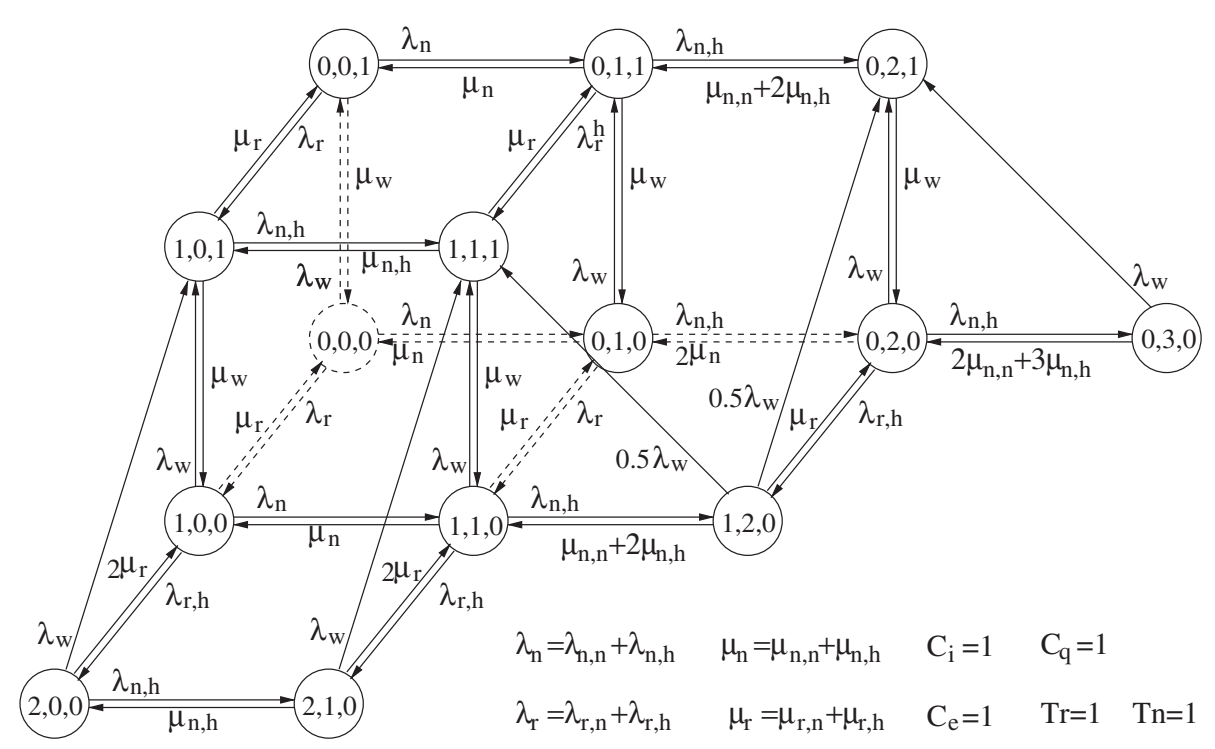

Figure $4 \mathrm{~A}$ state transition rate diagram for the threshold based channel reservation. 
state $(i, j, k)$, the nine indicator functions are described as follows.

The function $\delta_{r, n}(i, j, k)$, which is given in Equation (1), determines whether or not to admit a new real-time call into a cell in state $(i, j, k)$. If there is at least one free channel in state $(i, j, k)$ and the number of real-time calls is less than a threshold $T_{r}$, the new real-time call is admitted; otherwise, the real-time call is blocked. That is, if $i<T_{r}$ and $i+j+k<C$, the value of the function $\delta_{r, n}(i, j, k)$ is 1 ; otherwise, the value of the function $\delta_{r, n}(i, j, k)$ is 0 .

$$
\delta_{r, n}(i, j, k)= \begin{cases}1 & i<T_{r} \text { and } i+j+k<C \\ 0 & \text { otherwise. }\end{cases}
$$

The function $\delta_{n, n}(i, j, k)$ in Equation (2) determines whether or not to admit a new non-real-time call into a cell in state $(i, j, k)$. If the number of non-real-time calls in state $(i, j, k)$ is less than a threshold $T_{n}$, and there is at least one free channel in the state, the new non-real-time call is admitted; otherwise, the non-real-time call is blocked. That is, if $j<T_{n}$ and $i+j+k<C$, the value of the function $\delta_{n, n}(i, j, k)$ is 1 ; otherwise, the value of the function $\delta_{n, n}(i, j, k)$ is 0 .

$$
\delta_{n, n}(i, j, k)= \begin{cases}1 & j<T_{n} \text { and } i+j+k<C \\ 0 & \text { otherwise. }\end{cases}
$$

The function $\delta_{n, h}(i, j, k)$ in Equation (3) determines whether to accept a non-real-time inter-cell hand-off call in state $(i, j, k)$. A non-real-time inter-cell hand-of call is accepted in state $(i, j, k)$ if at least one free channel or free room in a queue exists in the state, i.e. $i+j+k<C+C_{q}$. That is, the value of the function $\delta_{n, h}(i, j, k)$ is 1 if $i+j+k<$ $C+C_{q}$. Otherwise, the inter-cell hand-off call is dropped; the value of the function $\delta_{n, h}(i, j, k)$ is 0 .

$$
\delta_{n, h}(i, j, k)= \begin{cases}1 & i+j+k<C+C_{q} \\ 0 & \text { otherwise }\end{cases}
$$

The function $\delta_{r, h}(i, j, k)$ in Equation (4) determines whether to accept a real-time inter-cell hand-off call in state $(i, j, k)$. A real-time inter-cell hand-off call is accepted in state $(i, j, k)$ under the following two situations. The first situation is that there is at least one free channel in a cell, i.e. $i+j+k<C$. On the contrary, if all channels are occupied (i.e. $i+j+k \geq C$ ), a real-time inter-cell hand-off call is also admitted as long as (i) there is at least one non-realtime user using a channel, i.e. $i+k<C$, and (ii) the queue in a cell is not full, i.e. $i+j+k<C+C_{q}$. This is because a non-real-time user will be preempted and placed into a queue, and then the released channel is allocated to the inter-cell hand-off call. Combining the conditions in the two situations, the value of the function $\delta_{r, h}(i, j, k)$ is 1 if $i+k<C$ and $i+j+k<C+C_{q}$; otherwise, the value of $\delta_{r, h}(i, j, k)$ is 0 .

$$
\delta_{r, h}(i, j, k)= \begin{cases}1 & i+k<C \text { and } i+j+k<C+C_{q} \\ 0 & \text { otherwise. }\end{cases}
$$

The function $\delta_{w}(i, j, k)$ in Equation (5) indicates whether a channel withdrawal is possible to occur in state $(i, j, k)$. If there is at least one leasehold channel in state $(i, j, k)$, a channel withdrawal may occur; i.e., the value of the function $\delta_{w}(i, j, k)$ is 1 if $0 \leq k<C_{e}$. Otherwise, no channel withdrawal will occur; i.e., the value of the function $\delta_{w}(i, j, k)$ is 0 if $k=C_{e}$.

$$
\delta_{w}(i, j, k)= \begin{cases}1 & 0 \leq k<C_{e} \\ 0 & \text { otherwise }\end{cases}
$$

A channel withdrawal may drop a call in state $(i, j, k)$, which can be classified into two situations: (i) there is no free capacity (no free channel and no free room in a queue), i.e. $i+j+k=C+C_{q}$, (ii) all channels are occupied by real-time calls, i.e. $i+k=C$. Except the two situations, a channel withdrawal does not drop a call. The function $\delta_{f}(i, j, k)$ in Equation (6) indicates whether the system is full (no free channel and no free room in a queue) in state $(i, j, k)$. If the system is full, i.e. $i+j+k=$ $C+C_{q}$, the value of $\delta_{f}(i, j, k)$ is 1 ; otherwise, the value of $\delta_{f}(i, j, k)$ is 0 . The function $\delta_{e}(i, j, k)$ in Equation (7) indicates whether real-time calls occupy (or exhaust) all channels except withdrawn channels in state $(i, j, k)$. The complements of the functions $\delta_{f}(i, j, k)$ and $\delta_{e}(i, j, k)$ are given in Equations (8) and (9).

$$
\begin{aligned}
& \delta_{f}(i, j, k)= \begin{cases}1 & i+j+k=C+C_{q}, \\
0 & \text { otherwise. }\end{cases} \\
& \delta_{e}(i, j, k)= \begin{cases}1 & i+k=C, \\
0 & \text { otherwise. }\end{cases} \\
& \bar{\delta}_{f}(i, j, k)= \begin{cases}1 & i+j+k<C+C_{q}, \\
0 & \text { otherwise. }\end{cases} \\
& \bar{\delta}_{e}(i, j, k)= \begin{cases}1 & i+k<C, \\
0 & \text { otherwise. }\end{cases}
\end{aligned}
$$

Global balance equations For a given eligible state $(i, j, k)$ in the state transition rate diagram of the threshold 
based channel reservation, the global balance equation is written as follows:

$$
\begin{aligned}
& P(i, j, k) \\
& =\frac{1}{G}\left\{\left[\lambda_{r, n} \delta_{r, n}(i-1, j, k)+\lambda_{r, h} \delta_{r, h}(i-1, j, k)\right] P(i-1, j, k)\right. \\
& +(i+1)\left(\mu_{r, n}+\mu_{r, h}\right) P(i+1, j, k) \\
& +\left[\lambda_{n, n} \delta_{n, n}(i, j-1, k)+\lambda_{n, h} \delta_{n, h}(i, j-1, k)\right] P(i, j-1, k) \\
& +\left[\min (j+1, C-i-k) \mu_{n, n}+(j+1) \mu_{n, h}\right] P(i, j+1, k) \\
& +\lambda_{w} \delta_{w}(i+1, j, k-1) \delta_{f}(i+1, j, k-1) \frac{i+1}{C-(k-1)} P(i+1, j, k-1) \\
& +\lambda_{w} \delta_{w}(i, j+1, k-1) \delta_{f}(i, j+1, k-1) \frac{C-(k-1)-i}{C-(k-1)} P(i, j+1, k-1) \\
& +\lambda_{w} \delta_{w}(i+1, j, k-1) \bar{\delta}_{f}(i+1, j, k-1) \\
& \times \delta_{e}(i+1, j, k-1) P(i+1, j, k-1) \\
& \left.+\lambda_{w} \delta_{w}(i, j, k-1) \bar{\delta}_{f}(i, j, k-1) \bar{\delta}_{e}(i, j, k-1)\right\}
\end{aligned}
$$

where

$$
\begin{aligned}
P(i, j, k)= \begin{cases}p(i, j, k) & i+j+k \leq C+C_{q} \\
0 & \text { otherwise. }\end{cases} \\
\begin{aligned}
G= & {\left[\left(\lambda_{r, n} \delta_{r, n}(i, j, k)+\lambda_{n, n} \delta_{n, n}(i, j, k)+\lambda_{r, h} \delta_{r, h}(i, j, k)\right.\right.} \\
& +\lambda_{n, h} \delta_{n, h}(i, j, k)+\lambda_{w} \delta_{w}(i, j, k) \\
& \left.+i\left(\mu_{r, n}+\mu_{r, h}\right)+\min (j, C-i-k) \mu_{n, n}+j \mu_{n, h}+k \mu_{w}\right]
\end{aligned}
\end{aligned}
$$

\section{Performance metrics}

We use the equilibrium probabilities of the Markov chain of the threshold based channel reservation scheme to calculate performance metrics as follows.

\section{Inter-cell hand-off dropping probability}

A real-time inter-cell hand-off call is dropped in state $(i, j, k)$ if the following two conditions are satisfied: (i) there is no free channel and (ii) there is no non-real-time call which can be preempted to release a channel; the latter condition occurs in a cell if there is no non-real-time user which is using a channel in the cell or the queue in the cell is full. Hence, the inter-cell hand-off dropping probability of real-time calls, $P_{r, c d}$, is the sum of the probabilities of the states $(i, j, k)$, where $i+k=C$ or $i+j+k=C+C_{q}$, which is as follow.

$$
P_{r, c d}=\sum_{i+k=C} p(i, j, k)+\sum_{\substack{i+k<C \\ i+j+k=C+C_{q}}} p(i, j, k) .
$$

In state $(i, j, k)$, a non-real-time inter-cell hand-off call is rejected from entering into a cell if the system is full (no free channel and no free space in a queue), i.e. $i+j+k=$ $C+C_{q}$. Therefore, the inter-cell dropping probability of non-real-time calls, $P_{n, c d}$, is the sum of the probabilities of the states $(i, j, k)$, where $i+j+k=C+C_{q}$, which is as follow.

$$
P_{n, c d}=\sum_{i+j+k=C+C_{q}} p(i, j, k) .
$$

Withdrawal dropping probability The probability that a real-time user is in state $(i, j, k)$ and suffers hand-off due to channel withdrawal is equal to $\frac{i}{C-k} p(i, j, k)$. Once the real-time user hand-offs due to channel withdrawal, the real-time call is dropped if there is no free channel and there is no non-real-time user which can be preempted to release a channel in state $(i, j, k)$. Therefore, the withdrawal dropping probability of real-time users, $P_{r, w d}$, can be obtained as follows.

$$
\begin{aligned}
& P_{r, w d}=\frac{1}{G_{r}} \sum_{k \neq C_{e}, i \geq 1}\left[\sum_{i+k=C} p(i, j, k)\right. \\
& \left.+\sum_{\substack{i+k<C \\
i+j+k=C+C_{q}}} \frac{i}{C-k} p(i, j, k)\right], \\
& \text { where } G_{r}=\sum_{\substack{k \neq C_{e}, i \geq 1 \\
i+j+k \leq C+C_{q}}} \frac{i}{C-k} p(i, j, k) \text {. }
\end{aligned}
$$

Similarly, the probability that a non-real-time user is in state $(i, j, k)$ and suffers hand-off due to channel withdrawal is equal to $\frac{C-k-i}{C-k} p(i, j, k)$. Once the non-real-time user hand-offs due to channel withdrawal, the non-realtime user is dropped if there is no free channel and no free room in the queue in state $(i, j, k)$. The channel withdrawal dropping probability of non-real-time calls, $P_{n, w d}$, is given as follows.

$$
\begin{gathered}
P_{n, w d}=\frac{1}{G_{n}} \sum_{\substack{k \neq C_{e}, i+k<C \\
i+j+k=C+C_{q}}} \frac{C-k-i}{C-k} p(i, j, k), \\
\text { where } G_{n}=\sum_{\substack{0 \leq k<C_{e} \\
i+j+k \leq C}} p(i, j, k) \frac{j}{C-k} \\
+\sum_{\substack{0 \leq k<C_{e} \\
C<i+j+k \leq C+C_{q}}} p(i, j, k) \frac{C-k-i}{C-k} .
\end{gathered}
$$

Mean waiting time We use Little's formula to derive the mean waiting time of non-real-time users. We first use steady state probabilities to derive the mean number 
of non-real-time users in a queue, $L_{q}$, which is given in Equation (17).

$$
L_{q}=\sum_{C<i+j+k \leq C+C_{q}}(i+j+k-C) p(i, j, k) .
$$

Then, using Little's formula, we can derive the average queuing time $W_{q}$ of non-real-time calls.

$$
W_{q}=L_{q} /\left(\lambda_{n, h}\left(1-P_{n, c d}\right)+\lambda_{p}\right)
$$

where $\lambda_{p}=$ rates of preempted non-real-time calls

$$
=\sum_{\substack{i+k<C \\ C \leq i+j+k<C+C_{q}}}\left(\lambda_{r, h}+\lambda_{w}\right) p(i, j, k) .
$$

Throughput The throughput, which is defined as the mean rate of completion calls in a cell. We first calculate the mean number of non-real-time calls which are being served in a cell. Then, the throughput of non-real-time calls $H_{n}$ is equal to the product of the mean number of non-real-time calls being served in a cell and the mean service rate of non-real-time calls. Similarly, we can derive the throughput of real-time calls $H_{r}$.

$$
\begin{aligned}
& H_{n}=\sum_{i+j+k \leq C+C_{q}} \min (j, C-i-k) p(i, j, k) \mu_{n, n} . \\
& H_{r}=\sum_{i+j+k \leq C+C_{q}} i p(i, j, k) \mu_{r, n} .
\end{aligned}
$$

Calculate performance metrics The steady state probability $p(i, j, k)$ in Equation (10) can be solved iteratively using the following procedure:

(1) Give initial values for $p(0,0,0)$ and $p(1,0,0)$, and set $P_{r, c d}$ and $P_{n, c d}$ to be zero.

(2) Use Equation (10) to calculate $p(i, j, k)$ for all eligible states $(i, j, k)$.

(3) Use Equations (13) and (14) to calculate $P_{r, c d}$ and $P_{n, c d}$, respectively.

(4) If (i) the different of the new $P_{r, c d}$ and the $P_{r, c d}$ in the previous iteration and (ii) the different of the new $P_{n, c d}$ and the $P_{n, c d}$ in the previous iteration are less than a small value $\epsilon$, the procedure is terminated. Otherwise, go to step 2.

The value of $\epsilon$ at Step 4 in the above procedure is selected to be $10^{-3}$. The iterative procedure converges, and we can obtain the equilibrium probabilities of eligible states, and use the equilibrium probabilities to calculate the remaining performance metrics in Equations (16)-(20).

\section{Performance evaluation}

In this section, we first verify numerical results by simulations. Then, we use an example to explain that the selected thresholds are optimal thresholds which satisfy the quality-of-service requirement while produce maximum throughput. Finally, we study the performances of real-time and non-real-time traffic at different loads with various withdrawal ratios. The parameters commonly used in the section are described as follows. The number of licensed channels in a cell, $C_{i}$, is 6 . The maximum number of leasehold channels in a cell, $C_{e}$, is equal to 24 . The mean lifetimes of real-time and non-real-time users, $\mu_{r, n}^{-1}$ and $\mu_{n, n}^{-1}$, are equal to $120 \mathrm{~s}$. The mean durations that realtime and non-real-time users sojourn in a cell, $\mu_{r, h}^{-1}$ and $\mu_{n, h}^{-1}$, are equal to $60 \mathrm{~s}$. The mean duration that leasehold channels are withdrawn, $\mu_{w}^{-1}$, is equal to $120 \mathrm{~s}$. The maximum number of calls which can be accommodated in a queue, $C_{q}$, is equal to 3 . The probability that new mobile users are real-time users, $p_{r}$, is equal to 0.6. The withdrawal ratio $\gamma$ is defined as the ratio of the mean number of withdrawal channels to the total number of leasehold channels in a cell. In this article, the Erlang load per channel is defined as the ratio of the new call arrival rate in a cell to the product of the mean number of available channels in a cell and the mean service rate of mobile users; that is, the Erlang load per channel is equal to $\lambda /(\bar{C} \bar{\mu})$, where $\bar{C}=C_{i}+(1-\gamma) C_{e}$ and $\bar{\mu}=p_{r} \mu_{r, n}+\left(1-p_{r}\right) \mu_{n, n}$. The hand-off rates of real-time and non-real-time users are two times the new call arrival rates of real-time and non-real-time users.

Event driven simulations are conducted to verify the numerical results. The simulation runs until a stopping criterion, which is the number of new calls is equal to $10^{6}$, is satisfied. Table 1 shows the numerical and simulation results in a cognitive network, in which the real-time and non-real-time thresholds are 30 , the withdrawal ratio is 0.2 and system load is 1 . From the table, we can observe that numerical and simulation results are similar.

We select optimal thresholds from a wide range of possible thresholds such that the quality of services are satisfied and throughput is maximized. The possible values of admission thresholds $T_{r}$ and $T_{n}$ range between 1 and 30. Quality-of-service metrics herein are the intercell hand-off dropping probability, channel withdrawal dropping probability and mean queuing time in a cell. The inter-cell hand-off dropping and channel withdrawal dropping probabilities will be kept below 0.04 . The mean waiting time of non-real-time users is set to be less than $1.0 \mathrm{~s}$. The quality-of-service metrics should be guaranteed when system load is 1 . Using the analysis, we could select the optimal real-time and non-real-time thresholds, $T_{r}=13$ and $T_{n}=30$, which guarantees the quality-ofservices when system load is 1 . In order to describe the 
Table 1 Analysis and simulation results

\begin{tabular}{llll}
\hline Performance metrics & & Analysis & Simulation \\
\hline New call blocking probability & Real-time & 0.28 & 0.29 \\
& Non-real-time & 0.28 & 0.29 \\
Inter-cell hand-off dropping probability & Real-time & 0.035 & 0.036 \\
& Non-real-time & 0.035 & 0.036 \\
Withdrawal dropping probability & Real-time & 0.044 & 0.045 \\
& Non-real-time & 0.033 & 0.034 \\
Queuing time (s.) & Non-real-time & 1.23 & 1.24 \\
Throughput (Erlang) & Real-time & 0.53 & 0.53 \\
& Non-real-time & 0.35 & 0.34 \\
\end{tabular}

selected thresholds are optimal, Table 2 shows the qualityof-services and throughputs of three combinations of realtime and non-real-time thresholds, $\left(T_{r}=14, T_{n}=30\right)$, $\left(T_{r}=12, T_{n}=30\right)$ and $\left(T_{r}=13, T_{n}=30\right)$. From the table, we can observe that the combinations $\left(T_{r}=\right.$ $\left.12, T_{n}=30\right)$ and $\left(T_{r}=13, T_{n}=30\right)$ guarantee qualityof-services, but the combination $\left(T_{r}=14, T_{n}=30\right)$ does not. In addition, the combination $\left(T_{r}=13, T_{n}=30\right)$ produces higher throughput than the combination $\left(T_{r}=\right.$ $12, T_{n}=30$ ). Based on the above observation, we can realize that the optimal thresholds are necessary because the optimal thresholds could produce the maximum throughput than the other combinations of thresholds under the guarantee of quality-of-services.

Cognitive networks may suffer different withdrawal ratios which may result in different system performances. In the following, we consider two withdrawal ratios, 0.4 and 0.8 , in a cognitive network where the length of queue is 5. The QoS requirement of queuing time is 5 seconds, and the remaining QoS requirements are 0.04. The optimal values of the admission thresholds $T_{r}$ and $T_{n}$ are 7 and
30 at $\gamma=0.8$; the optimal values of $T_{r}$ and $T_{n}$ at $\gamma=0.4$ are 30 and 30 .

Figures 5 and 6 show quality-of-service metrics on different withdraw ratios $\gamma$ at various loads. From the figures, we can observe two phenomena.

- The quality-of-service metrics at different withdrawal ratios satisfy our objective; that is, (i) the inter-cell hand-off and withdrawal dropping probabilities of real-time and non-real-time users are kept below 0.04, and (ii) the waiting time of non-real-time users are below 5 seconds.

- Non-real-time users produce lower inter-cell hand-off and withdrawal dropping probabilities than real-time users, because non-real-time users can be placed into a queue when channels are exhausted.

Figure 7 shows the throughputs of real-time and nonreal-time users. Since $60 \%$ of new calls are real-time users, the throughputs of real-time and non-real-time traffic will not exceed 0.6 and 0.4 , respectively. From the figure, we

Table 2 Comparison of QoS and throughput between optimal and non-optimal thresholds

\begin{tabular}{|c|c|c|c|c|}
\hline & & \multicolumn{2}{|c|}{ Non-optimal thresholds } & \multirow{2}{*}{$\begin{array}{l}\text { Optimal thresholds } \\
T_{r}=13, T_{n}=30\end{array}$} \\
\hline \multicolumn{2}{|c|}{ Performance metrics } & $T_{r}=14, T_{n}=30$ & $T_{r}=12, T_{n}=30$ & \\
\hline \multirow{2}{*}{$\begin{array}{l}\text { Inter-cell hand- } \\
\text { off dropping } \\
\text { probability }\end{array}$} & Real-time & 0.028 & 0.024 & 0.026 \\
\hline & Non-real-time & 0.028 & 0.024 & 0.026 \\
\hline \multirow{2}{*}{$\begin{array}{l}\text { Withdrawal } \\
\text { dropping } \\
\text { probability }\end{array}$} & Real-time & 0.037 & 0.032 & 0.034 \\
\hline & Non-real-time & 0.029 & 0.025 & 0.027 \\
\hline \multirow[t]{2}{*}{ Queuing time (s.) } & Non-real-time & 1.06 & 0.93 & 1.00 \\
\hline & Real-time & 0.50 & 0.47 & 0.48 \\
\hline \multirow{2}{*}{$\begin{array}{l}\text { Throughput } \\
\text { (Erlang) }\end{array}$} & Non-real-time & 0.36 & 0.37 & 0.37 \\
\hline & Total & 0.86 & 0.84 & 0.85 \\
\hline
\end{tabular}



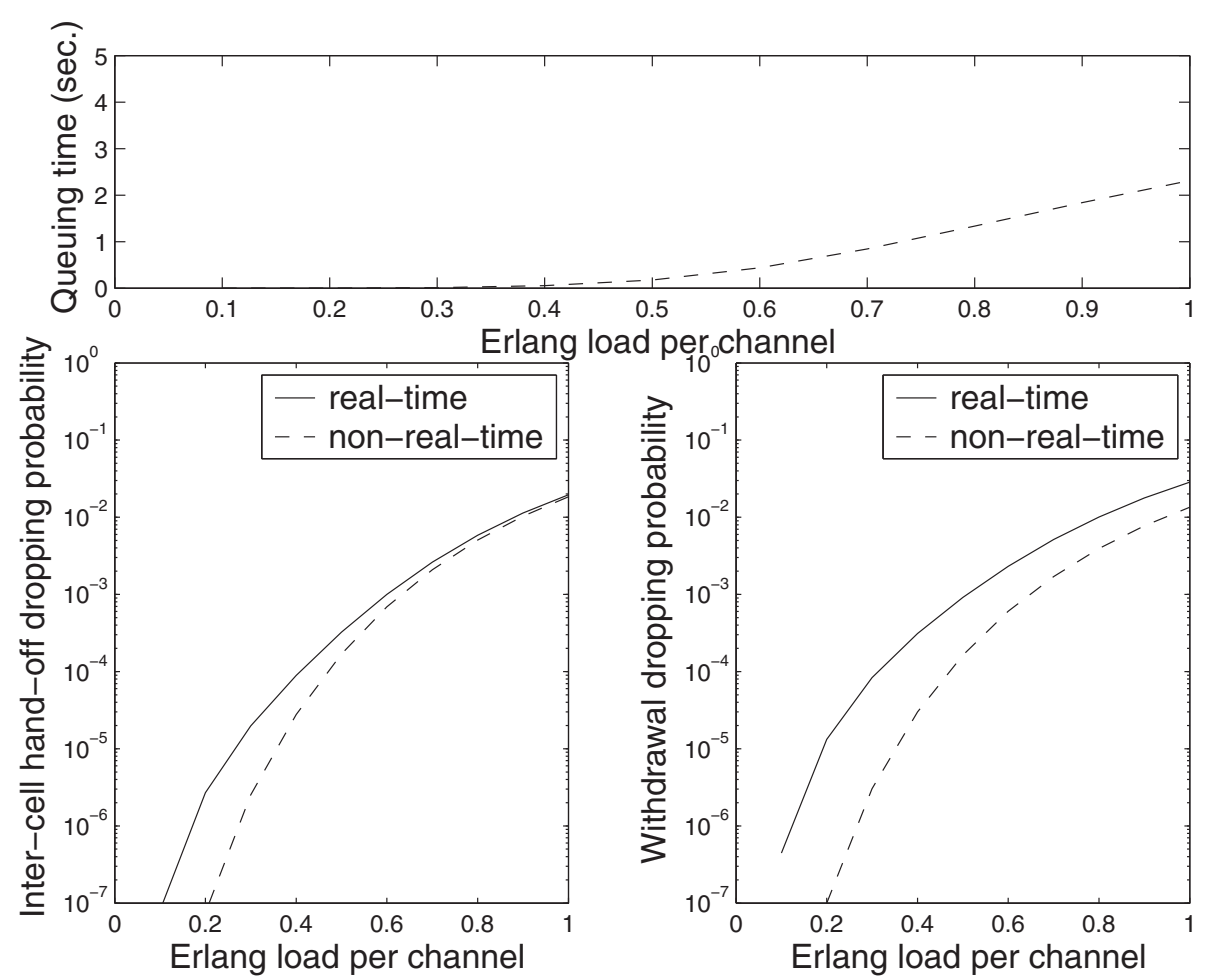

Figure 5 Quality-of-service metrics at $\gamma=0.4$.
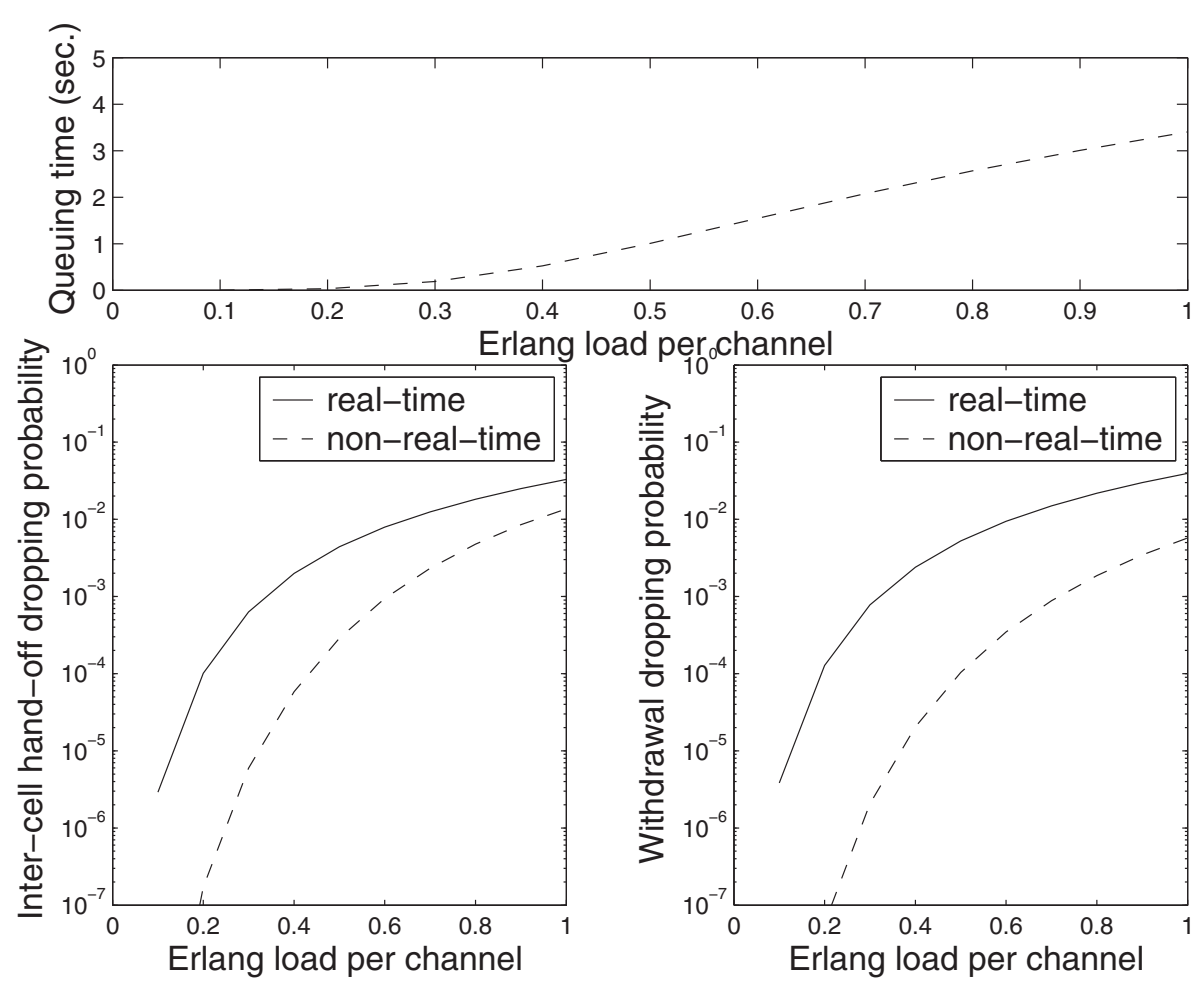

Figure 6 Quality-of-service metrics at $\gamma=0.8$. 

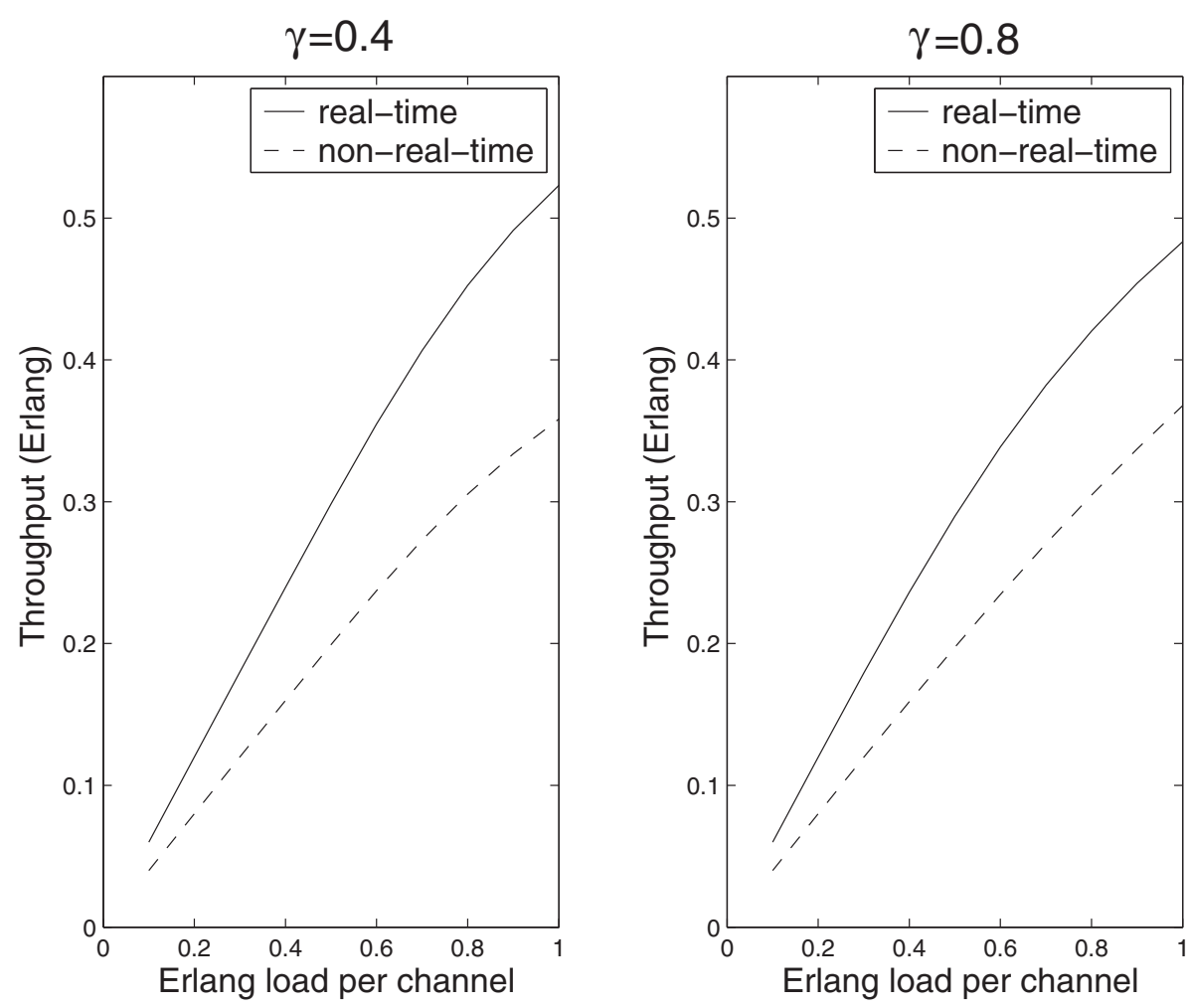

Figure 7 Throughput.

can observe that both real-time and non-real-time users produce high throughput under the constraint of qualityof-services. From the figure, we also observe that the throughput of real-time users at $\gamma=0.4$ is higher than that at $\gamma=0.8$ because more free channels are available to real-time users at lower withdrawal ratio. In addition, we observe that the throughputs of non-real-time users at $\gamma=0.4$ and $\gamma=0.8$ are similar because non-real-time users could be placed into a queue. Therefore, non-realtime users are less insensitive to the withdrawal ratio than real-time users.

\section{Conclusions}

Radio channel reservation is critical to provide qualityof-services for heterogeneous traffic in a cellular network which leases radio channels from another wireless networks. In such a network, we present a threshold-based channel reservation, which reserves channels in a single pool, for heterogeneous traffic, i.e. real-time and non-realtime traffic. We first develop a three-dimensional Markov chain to describe the system state of the threshold-based channel reservation in the network and present formal equilibrium equations of the Markov chain. Next, based on the Markov chain, we further derive the desired quality-of-service metrics (in terms of channel withdrawal dropping probability, inter-cell hand-off dropping probability, mean waiting time) and throughput. Then, given two thresholds for real-time and non-real-time traffic, we can apply our analyses to calculate the corresponding quality-of-services and throughput. Therefore, we can select optimal thresholds from a wide range of combinations of different thresholds such that the quality-ofservices of mobile users are satisfied while throughput is maximized. Numerical results show that, the selected thresholds can guarantee the quality-of-service requirements of real-time and non-real-time traffic at different situations of channel withdrawal and produce high throughput.

\section{Methods}

The numerical analysis of the Markov chain model in this paper is conducted by using $C$ programs. The simulations, which are used to verified the numerical analysis in this paper, are conducted by discrete event driven simulation with $\mathrm{C}$ programs.

\section{Competing interests}

The authors declare that they have no competing interests.

\section{Acknowledgements}

This research was partially supported by the National Science Council, Taiwan, under grants NSC 100-2221-E-017-007 and NSC 101-2221-E-017-011. 


\section{Author details}

${ }^{1}$ Department of Optoelectronics and Comm. Engineering, National Kaohsiung Normal University, Kaohsiung, 802 Taiwan. ${ }^{2}$ Department of Mathematics, National Kaohsiung Normal University, Kaohsiung, 802 Taiwan. ${ }^{3}$ Information and Comms. Research Lab., Industrial Technology Research Institute, Hsinchu 30043, Taiwan.

Received: 22 August 2011 Accepted: 27 May 2012

Published: 2 July 2012

\section{References}

1. FCC: ET Docket No 03-222 Notice of proposed rule making and order, December 2003

2. Q Zhao, BM Sadler, A survey of dynamic spectrum access. IEEE Signal Process. Mag. 24(3), 79-89 (2007)

3. IF Akyildiz, WY Lee, MC Vuran, S Mohantym, Next generation/dynamic spectrum access/cognitive radio wireless networks: a survey. Comput. Netw. 50(13), 2127-2159 (2006)

4. TA Weiss, FK Jondral, Spectrum pooling: an innovative strategy for the enhancement of spectrum efficiency. IEEE Commun. Mag. 42(3), s8-s14 (2004)

5. F Capar, I Martoyo, T Weiss, F Jondral, Comparison of bandwidth utilization for controlled and uncontrolled channel assignment in a spectrum pooling system. in Proceedings of the IEEE VTC, vol. 3, (Birmingham, Al, 2002), pp. 1069-1073

6. Y Xing, R Chandramouli, S Mangold, NS Shankar, Dynamic spectrum access in open spectrum wireless networks. IEEE J. Sel. Areas Commun. 24(3), 626-637 (2006)

7. X Zhu, L Shen, TSP Yum, Analysis of cognitive radio spectrum access with optimal channel reservation. IEEE Commun. Lett. 11(4), 304-306 (2007)

8. B Gavish, S Sridhar, Threshold priority policy for channel assignment in cellular networks. IEEE Trans. Comput. 46(3), 367-370 (1997)

9. T Kwon, S Kim, Y Choi, M Naghshineh, Threshold-type admission control in wireless/mobile multimedia networks using prioritized adaptive framework. IEEE Electron. Lett. 26(9), 852-854 (2000)

10. KW Ross, Multiservice Loss Models for Broadband Telecommunication Networks (Springer, Berlin, 1995)

11. SS Tzeng, Call admission control policies in cellular wireless networks with spectrum renting. Comput. Commun. 32(18), 1905-1913 (2009)

12. MH Ahmed, Call admission control in wireless networks: a comprehensive survey. IEEE Commun. Surv. Tutor. 7(1), 49-68 (2005)

doi:10.1186/1687-1499-2012-206

Cite this article as: Tzeng et al: Radio resource reservation for heterogeneous traffic in cellular networks with spectrum leasing. EURASIP Journal on Wireless Communications and Networking 2012 2012:206.

\section{Submit your manuscript to a SpringerOpen ${ }^{\circ}$ journal and benefit from:}

- Convenient online submission

- Rigorous peer review

- Immediate publication on acceptance

- Open access: articles freely available online

- High visibility within the field

- Retaining the copyright to your article

Submit your next manuscript at $\boldsymbol{~ s p r i n g e r o p e n . c o m ~}$ 